\title{
Designing optical-fiber modulators by using magnetic fluids
}

\author{
H. E. Horng,* J. J. Chieh, Y. H. Chao, and S. Y. Yang \\ Institute of Electro-Optical Science and Technology, National Taiwan Normal University, Taipei 116, Taiwan \\ Chin-Yih Hong \\ Department of Mechanical and Automation Engineering, Da-Yeh University, Chang-Hwa 515, Taiwan \\ H. C. Yang \\ Department of Physics, National Taiwan University, Taipei 106, Taiwan
}

Received July 26, 2004

\begin{abstract}
To reduce interface loss between optical fibers and devices in telecommunication systems, the development of an optical-fiber-based device that can be fused directly with fibers is important. A novel optical modulator consisting of a bare fiber core surrounded by magnetic fluids instead of by a $\mathrm{SiO}_{2}$ cladding layer is proposed. Applying a magnetic field raises the refractive index of the magnetic fluid. Thus we can control the occurrence of total reflection at the interface between the fiber core and the magnetic fluid when light propagates along the fiber. As a result, the intensity of the outgoing light is modulated by variation in field strength. Details of the design, fabrication, and working properties of such a modulator are presented. (C) 2005 Optical Society of America

OCIS codes: $\quad 060.2340,160.3820,230.4110$.
\end{abstract}

Because of properties such as ease of light-intensity manipulation and lower insertion loss, optical-fiber modulators have been widely used and integrated into optoelectronic systems. For example, an opticalfiber modulator can serve as an equalizer in a wavelength-division multiplexing system to equalize the amplified power of the channels. In addition, instead of tuning the bias current of distributed-feedback laser diodes, one uses an optical-fiber modulator to control the output power of light emitted from them. ${ }^{1}$ Because of their high value in these types of application, optical-fiber modulators have attracted a great deal of scientists' and engineers' interest, and different types of optical-fiber modulator are under development.

At present, the modulation mechanisms of opticalfiber modulators can be roughly categorized into two classes. $^{2}$ One is achieved by control of the physical disruption of the optical path through end separation, ${ }^{3}$ tilt misalignment, ${ }^{4-6}$ or lateral offset of the fibers. $^{3}$ The other is done by insertion of an absorbing or a partially reflective material, such as a liquid crystal $^{1}$ or a dicarbonylhydrazine ruthenium complex, ${ }^{7}$ into the optical path. Although these optical-fiber modulators have had a large effect on academic and industrial applications, they still suffer from a fatal disadvantage. This disadvantage is the insertion loss that results from the interface between the modulating block and the guiding fibers. Besides, from a manufacturing point of view the fabrication process is too complicated. Therefore, in this Letter we propose a novel type of optical-fiber modulator that consists of a bare fiber core surrounded by magnetic fluid.

Magnetic fluid is a colloid that contains magneticferrite nanoparticles dispersed in a liquid with the aid of a surfactant. Magnetic fluids are employed in electro-optical devices because of their versatile magnetically modulated optical properties,${ }^{8-11}$ especially that of a tunable refractive index. It was found previously that refractive index $n_{\mathrm{MF}}$ of magnetic fluid films increases linearly with the fluid concentration in a zero field, ${ }^{12}$ as plotted in Fig. 1(a). Furthermore, for a given fluid concentration, $n_{\mathrm{MF}}$ does not change until external magnetic field $H$ exceeds a certain strength; then $n_{\mathrm{MF}}$ becomes saturated at higher fields ${ }^{12}$ as shown in Fig. 1(b). The increase in $n_{\mathrm{MF}}$ in a higher field is attributed to the agglomeration of magnetic particles. In Fig. 1(b), the field modulated $n_{\mathrm{MF}}$ is shown for wavelengths of 632.8 and $1557 \mathrm{~nm}$. These results imply that we may utilize the tunable refractive index of a magnetic fluid as a cladding layer and control the occurrence of total reflection at the interface between the fiber's core and cladding magnetic fluid by varying the refractive index of the cladding layer through application of an external magnetic field. Thus the outgoing intensity of light through the modulator is assumed to be varied. In this Letter we investigate the feasibility of developing optical-fiber modulators by utilizing the magnetically tunable refractive index of a magnetic fluid. Details of the design, fabrication, and working properties of such a modulator are described.

The working principle of a magnetic-fluid-based optical-fiber modulator is shown schematically in Fig. 2. When a suitable concentration of magnetic fluid in which the zero-field $n_{\mathrm{MF}}$ is slightly smaller than that of $n_{\text {core }}$ of the fiber core for the wavelength used is selected, the guided light propagates within the fiber core owing to the total reflection at the interface between the core and the cladding magnetic fluid, as shown in Fig. 2(a). To select the fluid concentration properly, one has to know the refractive index of the 

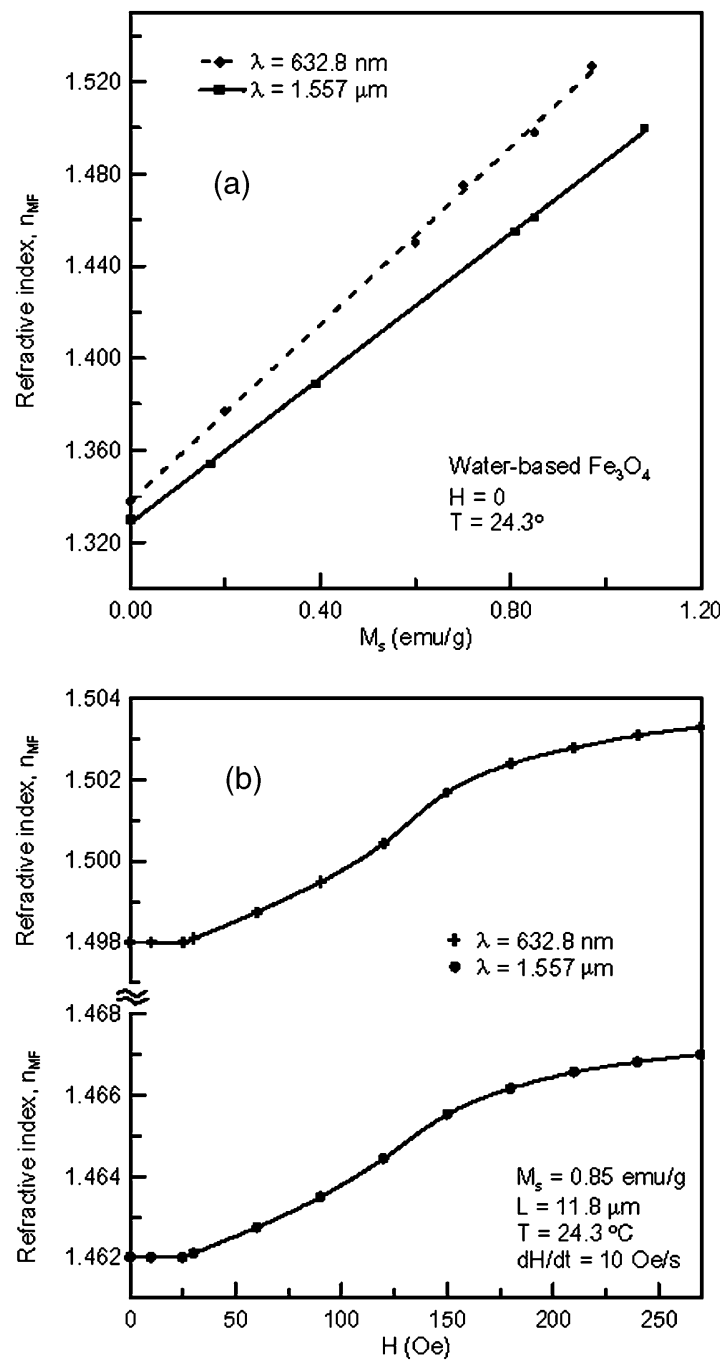

Fig. 1. (a) Concentration-dependent refractive index $n_{\mathrm{MF}}\left(M_{s}\right)$ of magnetic fluid in a zero field at $T=24.3^{\circ} \mathrm{C}$. (b) Magnetic-field-dependent refractive index $n_{\mathrm{MF}}(H)$ of a magnetic fluid film. Light with wavelengths of 632.8 and $1.557 \mu \mathrm{m}$ was used for both (a) and (b).

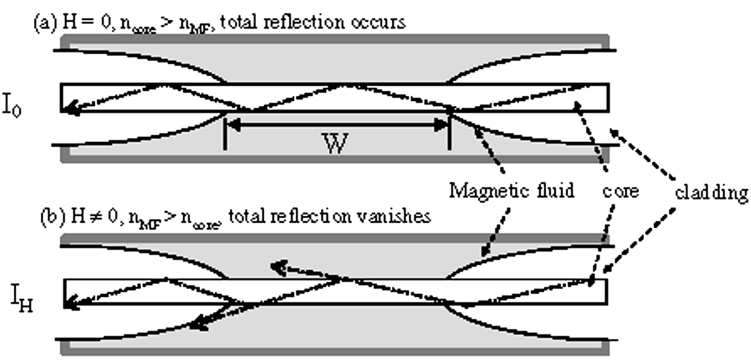

Fig. 2. Schematic illustration of the working principle of our optical-fiber magnetic-fluid modulator. Total reflection (a) occurs at zero field and (b) vanishes when an external field is applied. $W$ is the length of the bar-core section. $I_{0}$ and $I_{H}$, intensities transmitted through the modulator at zero and nonzero fields, respectively.

fiber core at the wavelength $\lambda$ used. For example, $\lambda$ in the experiment is $632.8 \mathrm{~nm}$. Thus $n_{\text {core }}$ is 1.46 . According to the result in Fig. 1(a), a magnetic fluid with a concentration of $0.61 \mathrm{emu} / \mathrm{g}$, where $\mathrm{emu}$ are electromagnetic units, is used. When a magnetic fluid field is applied, $n_{\mathrm{MF}}$ becomes larger than $n_{\text {core. }}$. The total reflection vanishes, and partial intensity of the guided light inside the fiber core is transmitted into the cladding layer, as illustrated in Fig. 2(b). As a result, the transmitted intensity is reduced when an external magnetic field is applied.

A schematic of the magnetic-fluid optical-fiber modulator is shown in Fig. 3(a). A multimode fiber with a core diameter of $62.5 \mu \mathrm{m}$ is used, and its cladding is etched away. The etched length is denoted $W$, and the image taken by an optical microscope for the fiber core is shown in Fig. 3(c). The fiber is fed through a capillary, and the capillary is filled with magnetic fluid. Then the magnetic fluid is sealed inside the capillary with UV glue. Thus the cladding layer is magnetic fluid along the bare-core section. Light of 632.8-nm wavelength is emitted from a $\mathrm{He}-\mathrm{Ne}$ laser and is coupled into the fiber through a lens. To remove the cladding modes of the propagating light along the optical fiber, another bare-core section is fabricated between the capillary and the incident end of the fiber. An image of this fiber-core section is shown in Fig. 3(d). Another bare-core section, whose image is shown in Fig. 3(b), is achieved between the capillary and the output end of the optical fiber to remove the cladding modes after the light passes through the bare core surrounded with magnetic fluid. It is worth noting that the core diameters of the three etched sections are smaller than that $(=62.5 \mu \mathrm{m})$ of the fiber core to make the magnetic fluid come into direct contract with the core. The output light's intensity through the magnetic-fluid-based optical-fiber modulator is detected by a photodetector. The magnetic field is provided with Helmholtz coils. Application of the field and the signal acquired from the photodiode is controlled automatically with a personal computer.

To demonstrate the feasibility of this optical modulator we coupled light of 632.8-nm wavelength into the optical-fiber modulator and detected the transmitted intensity with a photodiode. The bare-core section has a length $W$ of $417 \mu \mathrm{m}$ and is surrounded with 0.61-emu/g magnetic fluid. The refractive index of this magnetic fluid is smaller than that of the fiber core by 0.01 for a wavelength of $632.8 \mathrm{~nm}$. The modulation

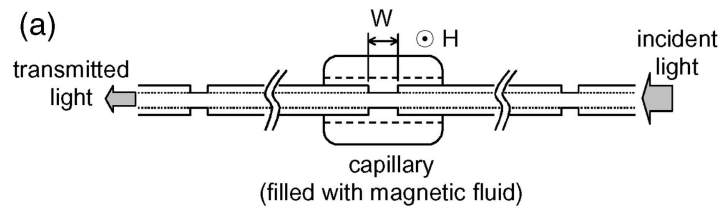

(b)

(c)

(d)

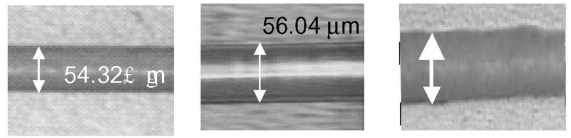

Fig. 3. (a) Schematic of the optical-fiber magnetic-fluid modulator. There are separate sections of bare cores; their images, taken by an optical microscope, are shown in (b) - (d). The middle section is sealed in a capillary field with magnetic fluid. External magnetic fluid is applied perpendicularly to the fiber. 


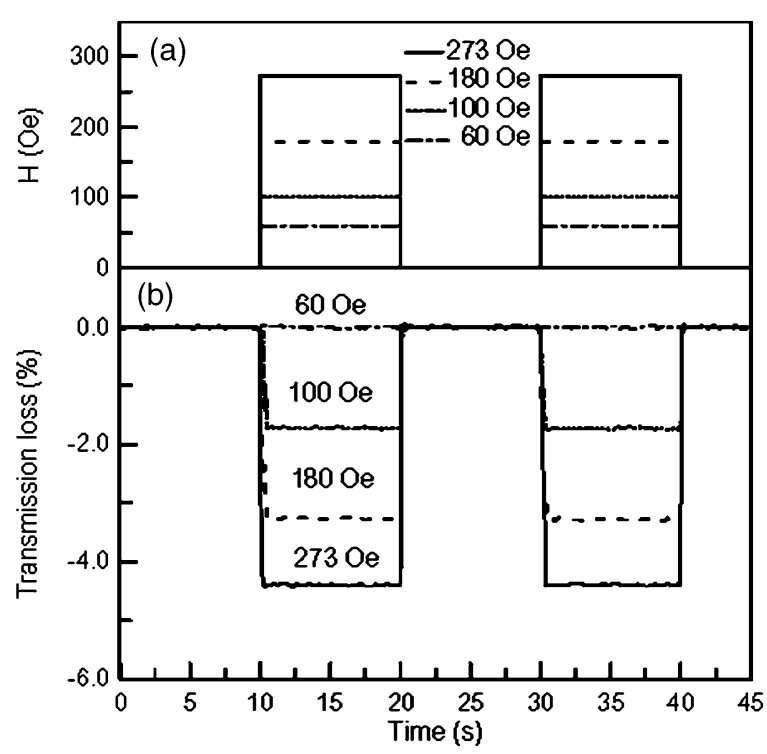

Fig. 4. Real-time variation in (a) applied magnetic fields and (b) transmission loss of the optical-fiber magnetic-fluid modulator in various fields.

characterization of the magnetic-fluid optical-fiber modulator was investigated experimentally, as shown in Fig. 4. The real-time square-wave-type variations in the applied magnetic fields are plotted in Fig. 4(a). The amplitudes were set as 60, 100, 180, and 273 Oe. The corresponding response of the transmission loss through the modulator to each external field was detected and is shown in Fig. 4(b). Here the transmission loss was obtained from $\left(I_{H}-I_{0}\right) / I_{0} \times 100 \%$, where $I_{0}$ and $I_{H}$ are the transmitted light intensity at zero and $H$ field strengths, respectively. We found no transmission loss below 60 Oe, but, when the amplitude of the magnetic field was increased, say, to $100 \mathrm{Oe}$, a transmission loss of $1.73 \%$ was observed when the external magnetic field was active. With increasing field amplitude, a higher transmission loss was achieved for the magnetic-fluid optical-fiber modulator. According to the results in Fig. 4(b), values of $3.28 \%$ and $4.4 \%$ were detected for transmission losses of the modulator at 180 and 273 Oe, respectively. The results shown in Fig. 4(b) clearly demonstrate the feasibility of developing optical modulators by utilization of the tunable refractive-index property of magnetic fluids. It is notable from Fig. 4(b) that, when the applied field is removed, the transmission loss returns to zero. This means that the intensity of light transmitted through the modulator recovers when the external field is off. The experimental data shown in Fig. 4(b) also exhibit good repetition of the magnetic-fluid optical-fiber modu- lator under operation. According to our experience, the rise time of a magnetic-fluid-based optical-fiber modulator when the external field is switched on or off is $\sim 10 \mathrm{~ms}$. This value may not be fast enough for high-speed applications but should be good for other applications, such as displays and routers. In addition, the transmission loss depends on the length of the bar-core section, the magnetic-field strength, and the number of modulators in a series. We tried to cascade two modulators, or elongate the bare-core section, to greatly increase the transmission loss by even more than $20 \%$. Hence, we believe that the desired modulation depth is achievable by careful selection of the cascading numbers, the bare-core section length, and the field strength.

In conclusion, by utilizing the tunable refractive index of a magnetic fluid, we designed and fabricated an optical-fiber modulator with a cladding layer of magnetic fluid. The experimental data on the transmission loss of the modulator exhibit its feasibility.

This research is supported by the National Science Council of Taiwan under grants NSC93-2112M-003-007, NSC93-2120-M-003-001, NSC93-2112M-003-017, and NSC92-2212-E-212-011 and in part by the Ministry of Education of the Republic of China under grant 91-N-FA01-2-4-2. H. E. Horng's e-mail address is phyfv001@scc.ntnu.edu.tw.

*Also with the Department of Physics, National Taiwan Normal University, Taipei 116, Taiwan.

\section{References}

1. K. Hirabayashi, M. Wada, and C. Amano, IEEE Photon. Technol. Lett. 13, 487 (2001).

2. S.-L. Chia, AMP J. Technol. 5, 19 (1996).

3. A. Benner, H. M. Presby, and N. Amitay, J. Lightwave Technol. 8, 7 (1990).

4. S. Sumriddetchkajorn and N. A. Riza, Opt. Commun. 205, 77 (2002).

5. N. A. Riza and S. Sumriddetchkajorn, Opt. Lett. 24, 282 (1999).

6. X. M. Zhang, A. Q. Liu, and C. Lu, J. Lightwave Technol. 21, 3417 (2003).

7. Y. H. Qi, P. Desjardins, X. S. Meng, and Z. Y. Wang, Opt. Mater 21, 255 (2002).

8. T.-S. Chin, Handbook of Magnetic Technologies (Taiwan Association for Magnetic Technology, Taipei, Taiwan, 2003).

9. P. Davies, J. Popplewell, and J. P. Llewellyn, IEEE Trans. Magn. 24, 1662 (1988).

10. H.-E. Horng, S. Y. Yang, S. L. Lee, C.-Y. Hong, and H. C. Yang, Appl. Phys. Lett. 79, 350 (2001).

11. F. Donatini, D. Jamon, J. Monin, and S. Neveu, IEEE Trans. Magn. 35, 4311 (1999).

12. S. Y. Yang, Y. F. Chen, H. E. Horng, C.-Y. Hong, W. S. Tse, and H. C. Yang, Appl. Phys. Lett. 81, 4931 (2002). 